\title{
Cultivation of early life history stages of Porphyra dioica from the British Isles
}

\author{
J. Knoop ${ }^{1}$ (1) • J. N. Griffin ${ }^{1}$ • S. Barrento ${ }^{1,2}$
}

Received: 3 June 2019 / Revised and accepted: 11 September 2019/Published online: 4 December 2019

(C) The Author(s) 2019

\begin{abstract}
Bladed Bangiales of the genus Porphyra/Pyropia are highly valuable red algae and extensively farmed in South East Asia. Interest is rising in cultivating species local to the North East Atlantic but the control of the heteromorphic life cycle of native species remains difficult as previous studies reported high inter- and intraspecific variability in required cultivation conditions. Here, working with Porphyra dioica from a UK source population, we conducted a series of experiments investigating the influence of substrate, temperature, photoperiod and light intensity on the development of early life history stages (conchocelis (filamentous sporophyte) and young thalli (gametophyte)). Special focus was the influence of temperature and photoperiod on mature conchocelis to induce a conchospore mass release - the current bottleneck of European Porphyra cultivation. Sporophytes grew largest on an oyster shell substrate and under long day conditions at $18^{\circ} \mathrm{C}$. A decrease in temperature from 18 to $9{ }^{\circ} \mathrm{C}$ initiated a mass conchospore release $\left(498 \pm 146\right.$ spores $\left.\mathrm{mL}^{-1}\right)$ from a $P$. dioica conchocelis culture grown in suspension. Released conchospores germinated into small thalli on nylon ropes, with best growth $\left(7.2 \pm 0.9 \%\right.$ day $\left.^{-1}\right)$ at low temperatures of $9^{\circ} \mathrm{C}$. Conchospore germination increased with decreasing light intensity but germination success was generally very low $(<5 \%)$, indicating the cultivation protocol needs further improvement. Our results reflect the adaptation of $P$. diocia to seasonal environmental conditions in temperate regions and the importance of these conditions for the successful cultivation. We are the first to describe a mass conchospore release for $P$. diocia growing in suspension which has important implications for commercial production.
\end{abstract}

Keywords Conchocelis $\cdot$ Conchospore $\cdot$ Conchosporangia $\cdot$ Aquaculture $\cdot$ Growth rate

\section{Introduction}

For centuries, marine macrophytes (seaweeds) have been used for human consumption, animal feed and fertiliser (Turner 2003; Dhargalkar and Pereira 2005). Global use has reached 30 million $\mathrm{t}$ (wet weight) with most biomass originating from extensive cultivation in South East Asia while production relies on harvesting in Europe (FAO 2018). Nearly 300,000 $\mathrm{t}$ is

Electronic supplementary material The online version of this article (https://doi.org/10.1007/s10811-019-01930-6) contains supplementary material, which is available to authorized users.

J. Knoop

jessica-knoop@web.de

1 Department of Biosciences, Swansea University, Singleton Park, Swansea SA2 8PP, UK

2 CIIMAR-Centre of Marine and Environmental Research, Terminal de Cruzeiros do Porto de Leixões, Av. General Norton de Matos s/n, 4450-208 Matosinhos, Portugal currently harvested in Europe and demand is expected to grow up to $10 \%$ annually, increasing pressure on local seaweed communities (Walsh and Watson 2012; Stagnol et al. 2013; Organic Monitor 2014; Capuzzo and McKie 2016; FAO 2018). In particular, traditionally consumed bladed Bangiales of the genus Porphyra/Pyropia are gaining popularity and acceptance as a sustainable and locally grown food ingredient as they are high in health beneficial substances such as antioxidants, minerals, vitamins and proteins (Burtin 2003; Ortiz et al. 2006; Cian et al. 2014, 2015; Mahadevan 2015; Mac Monagail et al. 2017). They retain high market prices as they are processed into valuable nori sheets, an indispensable ingredient in sushi, with a worldwide production worth nearly 2 billion US\$ (Levine and Sahoo 2010; FAO 2018). Furthermore, Porphyra has high nutrient uptake rates and the integration into existing shellfish or finfish cultivation sites could lower the environmental impact by reducing released nutrients and simultaneously add another valuable product to the cultivation site (Chopin et al. 2001; Chung et al. 2002; Kraemer et al. 2004; He et al. 2008; Pereira et al. 2008). 
Cultivation of Atlantic Porphyra species still faces several difficulties due to the complex heteromorphic life cycle that is currently not fully understood (Wang et al. 2006; Yan et al. 2007; Varela-Álvarez et al. 2018). The sexual life cycle (Fig. 1) alternates between the bladed gametophyte and the microscopic sporophyte (conchocelis) and is the major reproduction pathway for species in the North Eastern Atlantic (Brodie and Irvine 2003; Blouin et al. 2011). Male gametes (spermatia) are released from spermatangia and fertilise female gametes, resulting in zygotosporangia. Zygotospores are released from zygotosporangia and germinate upon settlement on suitable substrate (mollusc shells) into the filamentous conchocelis (sporophyte) stage (Drew 1949; Brodie and Irvine 2003). Conchospores are formed and released from conchosporangia in the sporophyte stage, thicker filaments developed by the conchocelis. Currently, the controlled mass release of conchospores from conchosporangia is a critical stage for cultivation - it is these spores that germinate into the edible thallus. This process is routinely done in Asian species with great success (Levine and Sahoo 2010), while the mass release of conchospores in European species has not been reported and forms a current bottleneck for largescale production.

The sporophyte and gametophyte require specific environmental conditions for their development, growth and maturation (Dring 1967; Sidirelli-Wolff 1992; Lu and Yarish 2011). A long photoperiod promotes vegetative growth in conchocelis native to the Northern Atlantic while a short photoperiod seems beneficial for conchosporangia production (Sidirelli-Wolff 1992; Orfanidis 2001; Pereira et al. 2004;
Varela-Alvarez et al. 2004; Holmes and Brodie 2005; He and Yarish 2006; Lu and Yarish 2011). A decrease in cultivation temperature triggers the mass conchospore release in Asian bladed Bangiales (Pyropia) and has also been demonstrated to initiate the release of conchospores in Northern Atlantic Porphyra species (Kornmann and Sahling 1991; Orfanidis 2001; Pereira et al. 2004; He and Yarish 2006; Levine and Sahoo 2010). In contrast, the gametophyte phase has been observed to grow well under various temperatures and photoperiods (Orfanidis 2001; Pereira et al. 2006; Green and Neefus 2015). Required temperature, photoperiod and light intensity for the life cycle completion probably depends on local conditions and might be an explanation for the observed limited success of the direct transfer of cultivation protocols between different species and populations (Orfanidis 2001; Lindstrom et al. 2008; Green and Neefus 2015). Controlling the development of the different life history stages in native species is crucial for a successful production on a larger scale and the focus of this study.

Porphyra dioica is a common species on rocky shores around the British Isles that is abundant throughout the year (Brodie and Irvine 2003; Holmes and Brodie 2004; Pereira et al. 2004). The possible year-round production makes it a promising candidate for seaweed cultivation around the British Isles. In this study, we investigated the development of early life history stages of $P$. dioica in a series of experiments with the aim to develop a cultivation protocol. We tested the (1) necessity of a shell substrate on the development of the sporophytic conchocelis phase, a method still used in commercial Pyropia cultivation in Asia since initiating a mass
Fig. 1 Sexual life cycle of Porphyra, alternating between the macroscopic thallus (gametophyte) and the microscopic conchocelis (sporophyte). The heteromorphic life cycle alternating between the haploid gametophyte and diploid sporophyte are currently under discussion, hence ploidy levels $(\mathrm{N})$ are included in brackets here

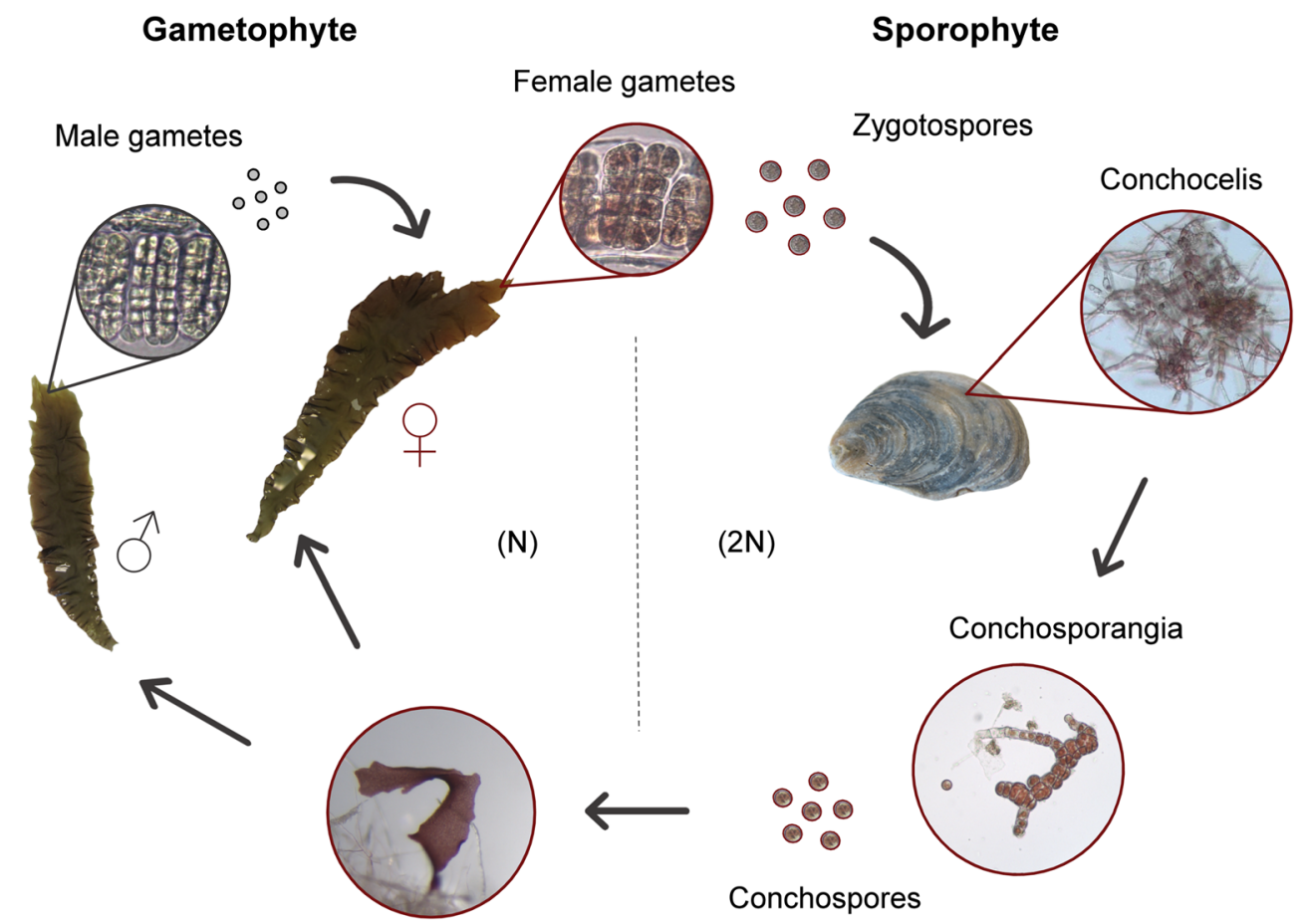


release of conchospores in free-floating conchocelis remains difficult (Levine and Sahoo 2010). Furthermore, we investigated the (2) effects of temperature and photoperiod on the development, growth and maturation of the conchocelis phase with the aim to induce a mass conchospore release from freefloating conchocelis. Finally, we evaluated (3) the influence of temperature, photoperiod and light intensity on the settlement and germination of conchospores on cultivation ropes as well as on growth and early development of the young thalli. We expected that high temperatures and a long photoperiod would be beneficial for conchocelis growth and that a conchospore mass release can be induced by a drop in temperature. The specific pigment constellations in red algae (especially phycobilins) are an adaptation to low light conditions and best growth of small thalli is therefore expected under lower light intensities.

\section{Materials and methods}

The following experiments focused on the development, growth and maturation of the sporophyte. Experiments are divided into three parts and addressed the different developmental stages of the sporophyte from (1) germination of released phyllospores, growth and maturation (we did not determine ploidy of released spores from the gametophytes and could not distinguish between agamospores and zygotospores which are both germinating into conchocelis; therefore, we used the proposed term phyllospores by Nelson et al. (1999) for released spores from the gametophyte with unknown ploidy), (2) conchospore release from conchosporangia and (3) germination and growth of conchospores into the gametophyte. All experiments were carried out in natural seawater originating from Swansea Bay, UK (N 51 $37^{\prime} 17.188^{\prime \prime}$, W $3^{\circ} 56^{\prime} 37.126^{\prime \prime}$ ), which was filtered down through a $11-\mu \mathrm{m}$ filter (Whatman filter No. 1) and autoclaved before being enriched with nutrients and vitamins (Provasolis 6, Miquel A\&B, Vitamin solution (Golven et al. 2014)). The growth medium was developed in order to form a matrix in the salt water providing a substrate for freefloating cultures. Germanium dioxide $\left(4 \mathrm{mg} \mathrm{L}^{-1}\right)$ was added to inhibit diatom growth (Markham and Hagmeier 1982). Experiments were carried out in constant temperature rooms $\left(15\right.$ and $18{ }^{\circ} \mathrm{C}$ ) or incubators $\left(9\right.$ and $12{ }^{\circ} \mathrm{C}$ ) equipped with fluorescent tubes (cool daylight, OSRAM L $36 \mathrm{~W} 865$ Lumilux). Due to the limited availability of independent temperature-controlled incubators, replicates per temperature treatment were spatially grouped. We are aware of the limitations of this design and therefore, results of the experiments with manipulating temperature should be considered with care. However, as the replicates were physically separated from each other in closed Erlenmeyer flasks and light intensity, light source, water and growth medium were identical, we are confident that the observed effects were a result of the manipulated temperature treatments. Temperature was constantly monitored by temperature loggers (DS1922L Thermochron iButton). Photoperiod and light intensity were manipulated within the same spatial area. Photoperiod was manipulated by separating different compartments within a shelving unit by black foil that was non-penetrable by light. Light intensity was manipulated by various layers of white nylon mesh to receive the desired light intensities as stated below. Photoperiods and light intensities differed between experiments and are stated in the sections below. Different photoperiod treatments could only be controlled in the constant temperature rooms $\left(15\right.$ and $\left.18{ }^{\circ} \mathrm{C}\right)$ due to spatial constraints. Light intensities differed slightly between different set ups but were equal within experiments and are not considered to impact the results (Orfanidis 2001; Pereira et al. 2004; Green and Neefus 2015; López-Vivas et al. 2015).

\section{Experiment 1: Phyllospore germination and conchocelis development}

\section{Effect of shell substrate}

The first experiment tested the necessity of a shell substrate on the germination of phyllospores (spores released from the gametophyte with unknown ploidy) and conchocelis growth. Phyllospores were obtained from a female Porphyra dioica individual collected in February 2017 at Langland, UK (N $51^{\circ} 34.016^{\prime \prime}$, W $\left.4^{\circ} 00.809^{\prime \prime}\right)$, a gently sloping sandy bay with extensive rocky parts and a well-established macrophyte community. The thallus was rinsed several times with sterile seawater and brushed gently with a sterile cotton wool to remove epiphytes. A small $0.5 \times 0.5 \mathrm{~cm}^{2}$ section of reproductive tissue was cut and rinsed again in sterile seawater before being placed overnight in darkness at $8{ }^{\circ} \mathrm{C}$ wrapped in a damp paper towel. Phyllospores were released upon re-immersion in sterile seawater. One thousand spores were transferred to $10 \times 10$ $\mathrm{cm}$ Petri dishes (Thermo Scientific Sterilin 100-mm Square Petri dishes) containing $30 \mathrm{~mL}$ of sterile seawater (final spore density 33 spores $\mathrm{mL}^{-1}$ ) enriched with nutrients and vitamins as stated above. Petri dishes either contained one oyster shell (collected from Swansea Bay, brushed and incubated in freshwater for $24 \mathrm{~h}$ followed by an incubation for $48 \mathrm{~h}$ at $60^{\circ} \mathrm{C}$ ) or just nutrient enriched seawater (Fig. 2, in Experiment 1 "Effect of shell substrate" section). The experiment was carried out in triplicate $(n=3)$. Spores were incubated at $18{ }^{\circ} \mathrm{C}$ and a neutral photoperiod (12:12 h light:dark). Light intensities between 10 and $100 \mu \mathrm{mol}$ photons $\mathrm{m}^{-2} \mathrm{~s}^{-1}$ have been shown to result in good germination and growth of the sporophyte with intensity not being a driving factor (Kornmann and Sahling 1991; Pereira et al. 2004; Lu and Yarish 2011). We therefore chose an irradiation of $50 \mu \mathrm{mol}$ photons $\mathrm{m}^{-2} \mathrm{~s}^{-1}$ which showed good results in germination and growth in 
Fig. 2 Schematic overview of the experiments conducted on phyllospore germination (Experiment 1), conchospore release (Experiment 2) and conchospore germination (Experiment 3 ) of $P$. dioica. All experiments were carried out in triplicate. Temperatures are stated in degrees Celsius and irradiance in $\mu \mathrm{mol}$ photons $\mathrm{m}^{-2} \mathrm{~s}^{-1}$. Photoperiods are defined as $\mathrm{SD}=$ short photoperiod $(08: 16 \mathrm{~h}$ light:dark), ND = neutral photoperiod $(12: 12 \mathrm{~h})$ and $\mathrm{LD}=$ long photoperiod (16:08 h). Shaded areas in tables indicate the treatment variable within an experiment

\section{Experiment 1 Phyllospore germination and conchocelis development}

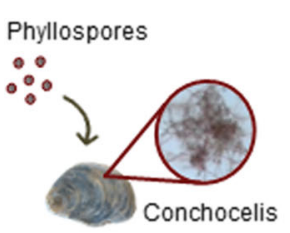

Effect of:
1.1 shell substrate

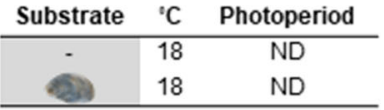

1.2 temperature

\begin{tabular}{cc}
${ }^{\circ} \mathrm{C}$ & Photoperiod \\
\hline $\mathbf{1 2}$ & $\mathrm{SD}$ \\
18 & $\mathrm{SD}$ \\
\hline
\end{tabular}

1.3 photoperiod

\begin{tabular}{c|c}
${ }^{\circ} \mathrm{C}$ & Photoperiod \\
\hline 18 & SD \\
18 & ND \\
18 & LD \\
\hline
\end{tabular}

\section{Experiment 2 Initiating conchospore mass release from conchosporangia}

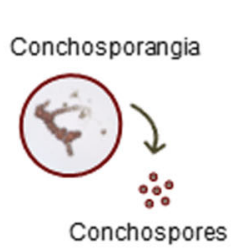

Effect of:
2.1 temperature

\begin{tabular}{cc} 
'C & Photoperiod \\
\hline 9 & SD \\
12 & SD \\
15 & SD \\
18 & SD \\
\hline
\end{tabular}

2.2 photoperiod

\begin{tabular}{c|c}
${ }^{\prime} \mathrm{C}$ & Photoperiod \\
\hline 18 & SD \\
18 & ND \\
18 & LD \\
\hline
\end{tabular}

\section{Experiment 3 Conchospore germination and development of young gametophytes}

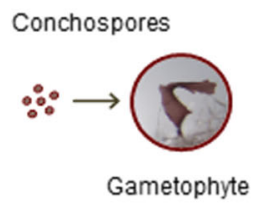

Effect of:

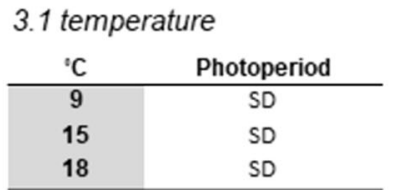

3.2 photoperiod

\begin{tabular}{c|c}
${ }^{\circ} \mathrm{C}$ & Photoperiod \\
\hline 15 & SD \\
15 & LD \\
15 & ND \\
\hline
\end{tabular}

\begin{tabular}{ccc}
\hline \multicolumn{3}{l}{3 light intensity } \\
Photoperiod & ${ }^{\circ} \mathrm{C}$ & Irradiance \\
\hline ND & 15 & $\mathbf{8 0}$ \\
ND & 15 & $\mathbf{1 3 0}$ \\
ND & 15 & $\mathbf{1 8 0}$ \\
\hline
\end{tabular}

preparative experiments. Germination into conchocelis and development was followed for 15 weeks. The medium was renewed after 2 weeks following experimental set up to ensure spore settlement and weekly thereafter. Pictures of all Petri dishes $(n=3)$ were taken by a Canon Powershot S90 and all visible colonies within each Petri dish were counted and measured at the end of the experiment by measuring the colony diameter processing the pictures with the imaging software ImageJ (1.51b, National Institute of Health, USA).

\section{Effect of temperature}

The second experiment tested the effect of different temperatures on phyllospore germination, conchocelis growth and development. Phyllospores were released from a female $P$. dioica individual collected from Langland in May 2017 following the same protocol as stated above. Round Petri dishes (Thermo Scientific Sterilin Standard $90 \mathrm{~mm}$ Petri Dishes) were filled with $30 \mathrm{~mL}$ of sterile nutrient enriched seawater and inoculated with 2300 phyllospores ( 77 spores $\mathrm{mL}^{-1}$ ) and closed with a lid to prevent desiccation and to minimise environmental impacts. Spore density differed from the previous experiment as more spores were released and therefore available for the experiment. Petri dishes were incubated at 12 and $18^{\circ} \mathrm{C}$ at a short photoperiod $(8: 16 \mathrm{~h}$ light:dark) and $50 \mu \mathrm{mol}$ photons $\mathrm{m}^{-2} \mathrm{~s}^{-1}$ in triplicate (Fig. 2, Experiment 1 the "Effect of temperature" section). Temperatures below $10{ }^{\circ} \mathrm{C}$ were observed to inhibit germination (Holmes and Brodie 2004; Pereira et al. 2004) while no conchocelis were observed above $20^{\circ} \mathrm{C}$ in preparative experiments. Nutrient enriched seawater was renewed first 2 weeks after inoculation to ensure attachment of spores and every week thereafter. Phyllospore germination, conchocelis growth and development were followed for 5 weeks. Pictures of all Petri dishes $(n=3)$ were taken by a stereo microscope (maximum $\times 63$ magnification) with an attached Leica camera (DFC 290) at the end of the experiment and all developed conchocelis colonies were measured and counted using the imaging software ImageJ. Conchocelis diameter and germination success (the percentage of developed conchocelis in relation to initial spore density) were compared at the end of the experiment. 


\section{Effect of photoperiod}

The third experiment tested the effect of photoperiod on phyllospore germination, conchocelis growth and development. The experimental design followed the setup as described under Experiment 1 the "Effect of temperature" section. The effect of photoperiod was tested by cultivating Petri dishes at a short (08:16 h dark:light), neutral (12:12 h) and long photoperiod $(16: 08 \mathrm{~h})$ at $18{ }^{\circ} \mathrm{C}$ and $50 \mu \mathrm{mol}$ photons $\mathrm{m}^{-2} \mathrm{~s}^{-1}$ in triplicate (Fig. 2, the "Effect of photoperiod" section).

\section{Experiment 2: Initiating conchospore mass release}

\section{Effect of temperature}

The fourth experiment tested the effect of temperature on the conchospore release from a free-floating conchocelis culture containing mature conchosporangia aiming to induce a mass conchospore release. The conchocelis culture was initiated from an individual collected at Langland in March 2016 (strain LL30/03/16, maintained at Swansea University at $18{ }^{\circ} \mathrm{C}$, a neutral photoperiod (12:12 light:dark) and $50 \mu \mathrm{mol}$ photons $\mathrm{m}^{-2} \mathrm{~s}^{-1}$ irradiance). The mature conchocelis culture was blended (IKA ULTRA-TURRAX T25 Basic S2) before $0.4 \mathrm{~g}$ fresh weight (fine scale, A\&D HR-120) was transferred to $100-\mathrm{mL}$ glass flasks filled with $100 \mathrm{~mL}$ sterile and nutrient enriched (as stated above) seawater. The stocking density was chosen as it is in the range of stocking densities commonly used in macroalgae cultivation (He and Yarish 2006; Pereira et al. 2006; Abreu et al. 2011; Zhong et al. 2016; Ashkenazi et al. 2018). To investigate the effect of temperature on the conchospore release, mature conchocelis were cultivated in triplicate for three weeks at $9,12,15$ and $18{ }^{\circ} \mathrm{C}$ under a short photoperiod (8:16 h light:dark) and $70 \mu \mathrm{mol}$ photons $\mathrm{m}^{-2} \mathrm{~s}^{-1}$ (Fig. 2, the Experiment 2 "Effect of temperature" section). Medium was renewed once after 15 days. Cultures were slightly aerated to ensure suspension of conchocelis in the water column. All cultures were checked every other day for released conchospores over a period of 3 weeks. Released conchospore density was determined by counting released conchospores in a $150 \mu \mathrm{L}$ sample under a light microscope $(\times 100$ magnifications). If spore density exceeded a density of 130 spores $\mathrm{mL}^{-1}$, spore density was determined in triplicate per replicate to minimise errors due to uneven distributed spores. Fresh weight was determined at the end of the experiment on a fine scale (A\&D HR-120). Days until peak conchospore release were identified and maximum conchospore density on that day calculated and compared between treatments.

\section{Effect of photoperiod}

To evaluate the effect of photoperiod on the conchospore release, cultures were incubated at $18{ }^{\circ} \mathrm{C}$ at a short $(\mathrm{SD}, 8: 16$ light:dark), neutral (ND, 12:12 light:dark) and long (LD, 16:8 light:dark) photoperiod and $70 \mu \mathrm{mol}$ photons $\mathrm{m}^{-2} \mathrm{~s}^{-1}$ in triplicate (Fig. 2, the "Effect of photoperiod" section). The experimental set up and sampling followed the design as previously described in Experiment 2 "Effect of temperature". The effect of photoperiod was tested at $18{ }^{\circ} \mathrm{C}$ to prevent adding an additional factor besides photoperiod as the conchocelis culture used in this experiment was cultivated at that temperature prior to the experiment.

\section{Experiment 3: Conchospore germination and development of young gametophytes}

\section{Effect of temperature}

This experiment aimed to investigate the effect of temperature on conchospore germination and growth of young thalli. One thousand five hundred released and free-floating conchospores (initiated from the strain LL30/03/16) were transferred to $100-\mathrm{mL}$ Erlenmeyer glass flasks (30 conchospores $\mathrm{mL}^{-1}$ ) filled with $50 \mathrm{~mL}$ sterile and nutrient enriched seawater as described before. Conchospore density used in this experiment was dependent by the amount of prior released conchospores. Each Erlenmeyer flask contained three 4.5 -cm-long nylon cultivation rope segments (Nylon Braid white $2 \mathrm{~mm}$, from www.ropeseller.co.uk) to test the attachment of conchospores on ropes for a possible out planting on an algae farm. To test the effect of temperature on conchospore germination and growth of young gametophytes, conchospores were incubated at 9,15 and $18{ }^{\circ} \mathrm{C}$ at a short photoperiod (08:16 h light: dark) and $80 \mu \mathrm{mol}$ photons $\mathrm{m}^{-2} \mathrm{~s}^{-1}$ (Fig. 2, Experiment 3 "Effect of temperature") in triplicate for 12 weeks. To refresh nutrients without losing unattached spores and to increase the cultivation volume, $50 \mathrm{~mL}$ sterilised and nutrient enriched seawater (as described previously) was added after 1 week. Medium was renewed weekly thereafter. Ropes were investigated weekly for settled and germinated spores. Small plantlets were measured 1 week after first appearance followed by every 2 weeks. Specific growth rates (SGR) were calculated (Yong et al. 2013) as:

$\operatorname{SGR}\left(\%\right.$ day $\left.^{-1}\right)=\left[\left(\frac{L_{t}}{L_{0}}\right)^{\frac{1}{t}}-1\right] \times 100$

where $L_{0}$ is the initial mean length and $L_{t}$ the final mean length per treatment, and $t$ the days of culture.

\section{Effect of photoperiod}

In this experiment, we tested the effect of photoperiod on conchospore germination and growth of young thalli. Porphyra dioica conchospores (1500 spores, initiated as 
stated previously) were transferred to $100-\mathrm{mL}$ glass Erlenmeyer flasks filled with $50 \mathrm{~mL}$ sterile and nutrient enriched seawater $\left(30\right.$ spores $\mathrm{mL}^{-1}$, constrained by amount of released conchospores) and one 10-cm-long rope as stated previously. The experiment was conducted in triplicate. $50 \mathrm{~mL}$ of nutrient enriched seawater were added 1 week after initiating the experiment to increase the water volume and refresh nutrients without losing remaining free-floating spores. Medium was renewed once per week thereafter. The effect of photoperiod on conchospore germination and development was tested at a short ( $8: 16 \mathrm{~h}$ light:dark), neutral (12:12 h light:dark) and long (16:8 h light:dark) photoperiod at $15^{\circ} \mathrm{C}$ and $130 \mu \mathrm{mol}$ photons $\mathrm{m}^{-2} \mathrm{~s}^{-1}$ (Fig. 2, Experiment 3 "Effect of photoperiod"). Conchospore germination success and SGR (1) of young thalli were followed over 11 weeks by counting and measuring all small plantlets visible under a stereo microscope $(\times 63$ maximum magnification).

\section{Effect of light intensity}

The final experiments tested the effect of light intensity on conchospore germination and growth of young thalli (Fig. 2, "Effect of light intensity"). The experimental design and sampling followed the description under Experiment 3 "Effect of photoperiod". The effect of light intensity on conchospore germination and development was investigated by incubating conchospores as described previously (Experiment 3 "Effect of photoperiod") under a low $\left(80 \mu \mathrm{mol}\right.$ photons $\left.\mathrm{m}^{-2} \mathrm{~s}^{-1}\right)$, medium $\left(130 \mu \mathrm{mol}\right.$ photons $\left.\mathrm{m}^{-2} \mathrm{~s}^{-1}\right)$ and high $(180 \mu \mathrm{mol}$ photons $\left.\mathrm{m}^{-2} \mathrm{~s}^{-1}\right)$ light intensity at $15^{\circ} \mathrm{C}$ and a neutral photoperiod (12:12 h).

\section{Statistical analysis}

All results are reported and visualised as group arithmetic means \pm standard errors. The amount of conchocelis colonies growing with or without a shell substrate (Effect of shell substrate) was compared by a two-sample $t$ test. Conchocelis colony size with or without a shell substrate (Effect of shell substrate) were analysed by a non-parametric Mann-Whitney $U$ test. A twosample $t$ test was used to test the influence of temperature on phyllospore germination and conchocelis development (Effect of temperature). The effect of photoperiod on phyllospore germination and conchocelis development (Effect of photoperiod) was analysed by analysis of variance (ANOVA) followed by post hoc Tukey tests after assumptions for normality and homogeneity were assured. The effect of temperature on timing of the peak conchospore release (Effect of temperature) was analysed by a non-parametric Kruskal-Wallis test due to the heteroscedasticity of the data. The effect of photoperiod on the time after which the peak conchospore release was observed (Effect of photoperiod) was analysed by ANOVA as previously described. Conchospore densities (Experiment 2) were square root transformed to meet
ANOVA assumptions before the effect of temperature or photoperiod on released conchospore densities were analysed by ANOVA. Results of the conchospore germination experiments (the "Effect of temperature," "Effect of photoperiod," and "Effect of light intensity" sections) were analysed using a linear Mixed Model approach with the nlme package in R (Pinheiro et al. 2019), to account for the repeated measures design.

\section{Results}

\section{Experiment 1: Phyllospore germination and conchocelis development}

\section{Effect of shell substrate}

Phyllospores germinated in all treatments into filamentous conchocelis (sporophyte) colonies growing attached to the bottom of the Petri dishes or on top of the oyster shells. Conchocelis filaments were first visible on the bottom of the Petri dishes after 2 weeks. Germinated phyllospores were difficult to see on the oyster shells and were spotted after 3 weeks under the stereomicroscope with a $\times 64$ magnification. More conchocelis colonies were visible on the Petri dish bottom compared with the oyster shell surface $(p<0.001)$. Percentage germination success on oyster shells containing treatments was $3.3 \pm 0.7 \%$ (mean $\pm \mathrm{SE}$ ) and $11.4 \pm 0.3 \%$ in Petri dishes without shells (Fig. 3a).

Conchocelis grew in all treatments but differed significantly in size between substrates. Conchocelis colonies growing on oyster shells grew within the shell matrix and were much larger in diameter $(p<0.001)$ compared with colonies growing on Petri dish bottoms (Fig. 3b). Colonies on oyster shells a)

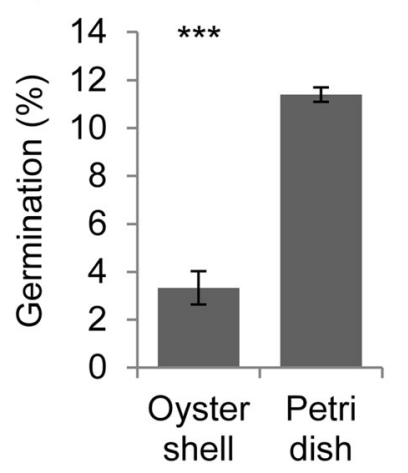

b)

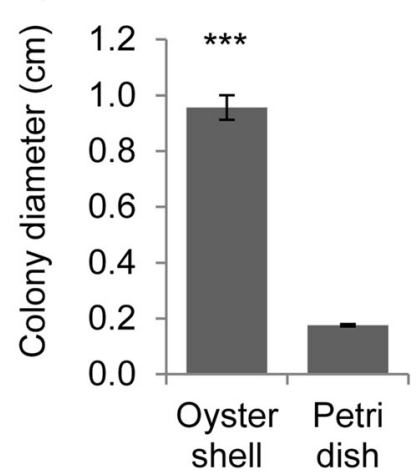

Fig. 3 Porphyra dioica percentage phyllospore germination success a and conchocelis diameter $\mathbf{b}$ after 15 weeks of cultivation in $10 \times 10-\mathrm{cm}$ Petri dishes with and without an oyster shell substrate cultivated at $18^{\circ} \mathrm{C}$, a neutral photoperiod (12:12 h light:dark) and $50 \mu \mathrm{mol}$ photons $\mathrm{m}^{-2} \mathrm{~s}^{-1}$ in $30 \mathrm{~mL}$ sterile and nutrient enriched seawater (Experiment 1 the "Effect of shell substrate" section). Shown are means \pm standard errors (replicates $n=3$ ). Triple asterisks indicate significant differences with $p<0.001$ 
were $0.96 \pm 0.04 \mathrm{~cm}$ while conchocelis growing on Petri dish bottoms were $0.18 \pm 0.004 \mathrm{~cm}$ in diameter. Morphology differed also between colonies grown on oyster shells or Petri dish bottoms. Colonies growing on oyster shells were much more spread compared with colonies growing on Petri dish bottoms which were growing as tufts. Conchosporangia developed in conchocelis growing on oyster shells and Petri dish bottoms after 8 weeks. However, conchosporangia were concentrated in the centre of colonies on oyster shells and growing outside of the shell in contrast to most conchocelis filaments that grew within the shell matrix. Conchosproangial filaments growing in colonies attached to Petri dish bottoms however developed within colony tufts. Conchospores were not observed in any treatments over the experimental period.

\section{Effect of temperature}

Phyllospores germinated into conchocelis (sporophyte) under all temperature treatments tested (Fig. 4a, b), growing attached to the Petri dish bottoms. However, a significantly higher amount $(p<0.01)$ of phyllospores germinated into the conchocelis phase cultivated under $18{ }^{\circ} \mathrm{C}$ (Fig. 4a). Conchocelis diameter also varied between temperature treatments at the end of the incubation period and temperatures of $18{ }^{\circ} \mathrm{C}$ resulted in larger conchocelis $(1.0 \pm 0.2 \mathrm{~mm})$ colonies (Fig. 4b) compared with the $12{ }^{\circ} \mathrm{C}$ treatment $(0.3 \pm 0.1 \mathrm{~mm})$.

\section{Effect of photoperiod}

Phyllospores germinated under all tested photoperiods (Fig. $4 c, d$ ) with a higher germination success incubated at a short or neutral photoperiod. Cultivation at a long photoperiod resulted in a lower germination success compared with short or neutral photoperiods $(p<0.05)$. A long photoperiod resulted in largest colonies $(1.3 \pm 0.2 \mathrm{~mm}$ in diameter). Conchosporangia started to grow at the end of the experiment under a short photoperiod.

\section{Experiment 2: Initiating conchospore mass release}

\section{Effect of temperature}

Conchospores were released under all tested temperature treatments with no significant effect of temperature on the peak release of conchospores (Fig. 5a). Variability was very high under cultivation at $12{ }^{\circ} \mathrm{C}$ where one replicate showed a peak conchospore release only after 19 days of incubation. We noticed the trend that conchospores were released sooner when cultures were transferred to $9{ }^{\circ} \mathrm{C}$ and a short photoperiod $(5.3 \pm 0.3$ days $)$. The amount of released conchospores decreased with increasing cultivation temperatures $(p<0.05)$ and was highest $\left(498 \pm 146\right.$ spores $\left.\mathrm{mL}^{-1}\right)$ at cultivation under $9{ }^{\circ} \mathrm{C}$ and a short photoperiod (Fig. 5b). If related to original conchocelis/conchosporangia biomass used in the experiment, the measured conchospore release corresponds to 1.5 million conchospores per g DW conchocelis/conchosporangia culture. The number of suspended spores decreased after the observed peak release. Only very few spores $(129 \pm 31$ spores $\mathrm{mL}^{-1}$ ) were released when cultivated at $18^{\circ} \mathrm{C}$. Free-floating spores were visible for a longer period in the $15^{\circ} \mathrm{C}$ compared with the 9 and $12{ }^{\circ} \mathrm{C}$ treatments.

\section{Effect of photoperiod}

Conchospores were released under all tested photoperiods at $18^{\circ} \mathrm{C}$ and $70 \mu \mathrm{mol}$ photons $\mathrm{m}^{-2} \mathrm{~s}^{-1}$. However, photoperiod
Fig. 4 Effect of temperature (a, $\mathbf{b}$, Experiment 1 the "Effect of temperature" section) and photoperiod (c, d, Experiment 1 the "Effect of photoperiod" section) on Porphyra dioica phyllospore germination and conchocelis diameter after a 5 week incubation at 12 or $18^{\circ} \mathrm{C}$ temperatures and different photoperiods $(8: 8,12: 12,16: 8 \mathrm{~h}$ light:dark) at $50 \mu \mathrm{mol}$ photons $\mathrm{m}^{-2} \mathrm{~s}^{-1}$. Shown are means \pm standard errors (replicates $n=3$ ). Significant differences are either indicated by stars $\left(^{* *} p<0.005\right.$, $* * * p<0.001)$ or by small letters indicating differences between treatments with $p<0.05$ ) a)

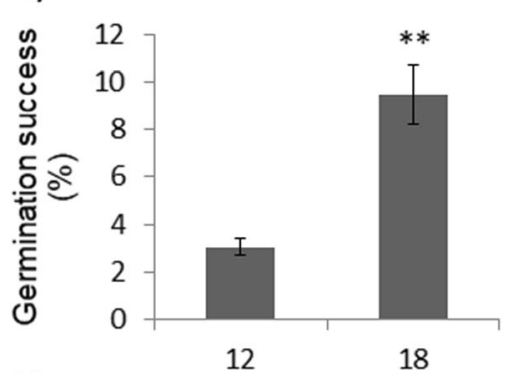

b)

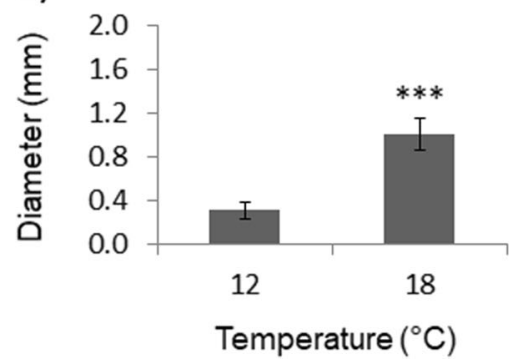

c)
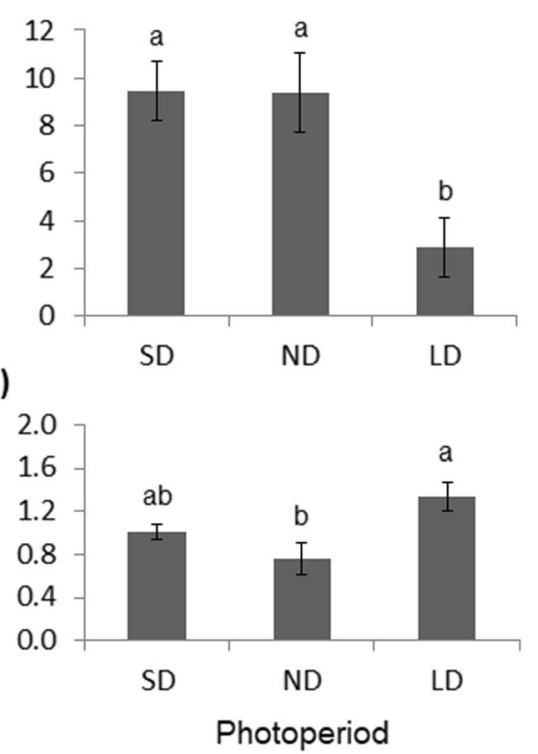
Fig. 5 Effect of temperature (Experiment 2 the "Effect of temperature" section) until Porphyra dioica conchospore peak release was observed (a) and amount of released conchospores (b) during the peak release in $P$. dioica at different temperatures cultivated under a short photoperiod (8:16 h light:dark) at $80 \mu \mathrm{mol}$ photons $\mathrm{m}^{-2} \mathrm{~s}^{-1}$. Shown are means \pm standard error (sampling size $n=3$ ). Small letters indicate significant differences a)

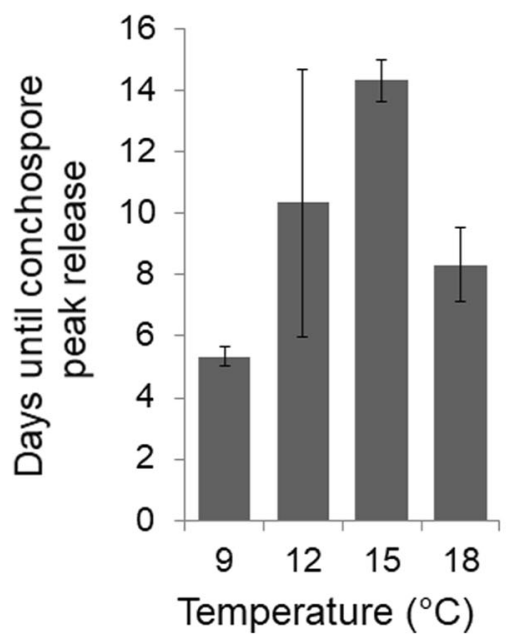

b)

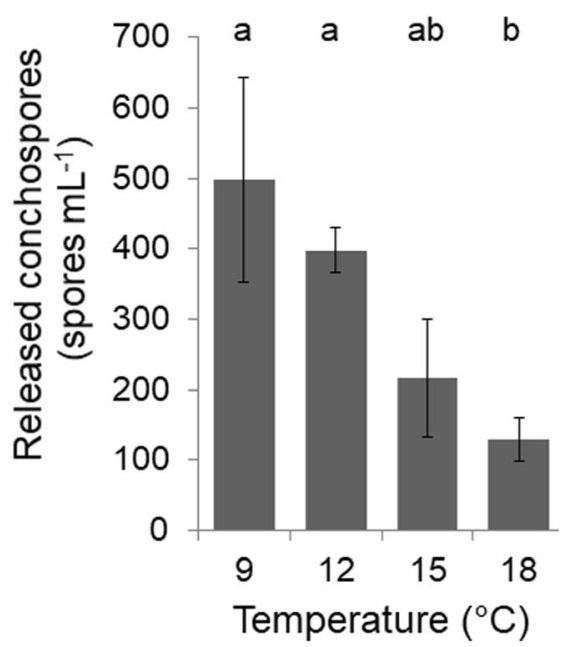

had no effect on time until a peak release of $P$. dioica conchospores occurred (Supplementary Material 1). Peak conchospore release was observed after on average $8.5 \pm$ 1.0 days. Furthermore, photoperiod did not affect the amount of released conchospores during the peak release, on average $95 \pm 20$ spores $\mathrm{mL}^{-1}$.

\section{Experiment 3: Conchospore germination and development of young gametophytes}

\section{Effect of temperature}

Conchospores germinated into the thallus (gametophyte) phase under all tested conditions. Germination success, however, was very low (Fig. 6a) with no effect of temperature. Conchospores that germinated into thalli increased continuously in size over the 12 weeks experimental period (Fig. 6 b). Thalli were largest cultivated at $15^{\circ} \mathrm{C}$ for the first 8 weeks of incubation compared with thalli cultivated at 9 or $18{ }^{\circ} \mathrm{C}$ $(p<0.05)$. After 8 weeks however, thalli incubated at $9{ }^{\circ} \mathrm{C}$ increased quickly in size and were largest $(19.6 \pm 0.4 \mathrm{~mm})$ after 12 weeks of cultivation compared with thalli grown at $15^{\circ} \mathrm{C}(9.3 \pm 1.3 \mathrm{~mm})$ or $18^{\circ} \mathrm{C}(5.6 \pm 0.4 \mathrm{~mm})$.

Specific growth rate (SGR) of young thalli (Fig. 6c) was significantly influenced by cultivation temperatures $\left(\chi^{2}(2)=\right.$ $8.07, p=0.02)$. Initially, SGR of thalli was highest (12.2 \pm $0.8 \%$ day $\left.^{-1}\right)$ if cultivated at $15{ }^{\circ} \mathrm{C}$ and lowest $(7.3 \pm 1.4 \%$ day $^{-1}$ ) when cultivated at $18^{\circ} \mathrm{C}$. Over the experimental timeline of 12 weeks however, SGR in young thalli cultivated at $15{ }^{\circ} \mathrm{C}$ decreased steadily to $3.2 \pm 0.7 \%$ day $^{-1}$ from week eight onwards. SGR of thalli cultivated at $18^{\circ} \mathrm{C}$ also decreased from initial $7.3 \pm$ $1.1 \%$ day $^{-1}$ to $3.2 \pm 0.7 \%$ day $^{-1}$. Thalli cultivated at $9{ }^{\circ} \mathrm{C}$ grew only slower in the third week before maintaining constant growth at $7.2 \pm 0.9 \%$ day $^{-1}$ and was higher at the end of the experiment compared with SGR of thalli grown at 15 or $18{ }^{\circ} \mathrm{C}$.

\section{Effect of photoperiod}

P. dioica conchospores germinated into small thalli (gametophytes) under all photoperiods (short day (SD 8:16 h light:dark), neutral day (ND 12:12 h light:dark) and long day (LD 16:8 h light:dark)) tested. Photoperiod had no effect on conchospore germination success or growth of young thalli (supplementary material Table S1, Fig. S2).

\section{Effect of light intensity}

In contrast to photoperiod, light intensity influenced the germination success $\left(\chi^{2}(2)=12.9, p<0.005\right)$ of $P$. dioica conchospores. However, germination success was generally very low as observed in the previous experiments (Fig. 7a). Highest germination success $(1.3 \pm 0.2 \%)$ was observed when exposed to $80 \mu \mathrm{mol}$ photons $\mathrm{m}^{-2} \mathrm{~s}^{-1}$ compared with higher light intensities. Germination success did not differ between the medium and high light intensity treatment. Small thalli that germinated from conchospores increased in size over the experimental period in all light intensities tested (Fig. 7b). Light intensity affected size of thalli $\left(\chi^{2}(2)=11.1, p<0.005\right)$ and a medium light intensity of $130 \mu \mathrm{mol}$ photons $\mathrm{m}^{-2} \mathrm{~s}^{-1}$ resulted in longest thalli $(3.2 \pm 0.4 \mathrm{~cm})$. Shortest individuals $(0.9 \pm$ $0.3 \mathrm{~cm}$ ) were observed under $80 \mu \mathrm{mol}$ photons $\mathrm{m}^{-2} \mathrm{~s}^{-1}$. The larger size under $130 \mu \mathrm{mol}$ photons $\mathrm{m}^{-2} \mathrm{~s}^{-1}$ was mainly driven by the increase in size between weeks 7 and 9. Light intensity had no effect on the overall SGR.

\section{Discussion}

Our results show that early life history stages of Porphyra dioica from South Wales are strongly impacted by temperature, photoperiod and light intensity, reflecting the adaptation 
Fig. 6 Influence of temperature (Experiment 3 the "Effect of temperature" section) on Porphyra dioica conchospore germination success into the thallus phase (a), size development (b) and specific growth rates (SGR) (c) of small $P$. dioica thalli growing on nylon ropes at a short photoperiod (8:16 h light:dark) and $80 \mu \mathrm{mol}$ photons $\mathrm{m}^{-2} \mathrm{~s}^{-1}$ for 12 weeks in triplicates $(n=3)$. Examples, of with small thalli and conchocelis overgrown nylon ropes, are represented (d). Temperature significantly influenced small thalli size and SGR $(p<0.05)$ with largest thalli and fastest growth observed at $9{ }^{\circ} \mathrm{C}$. Shown are group means \pm standard errors a)

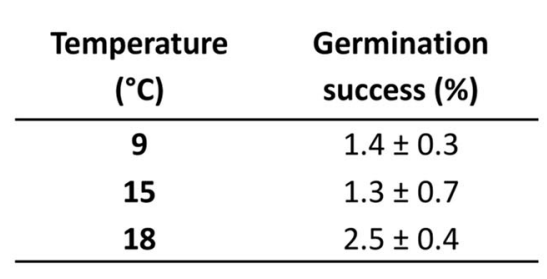

b)

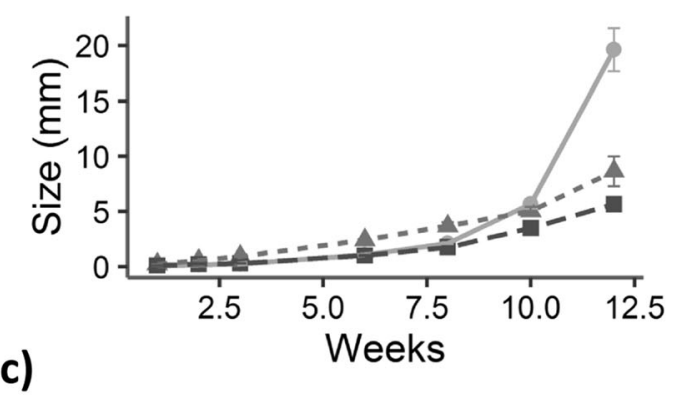

c)

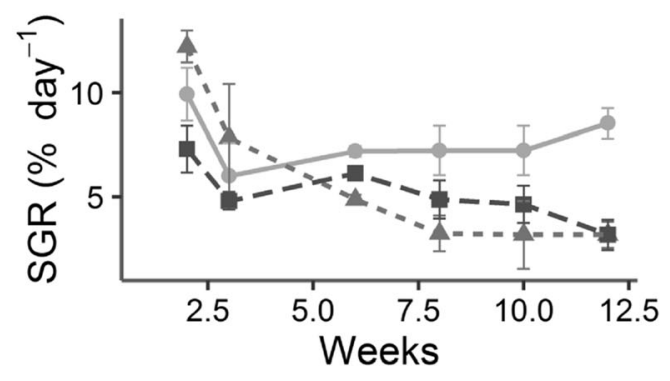

Temperature $\rightarrow 9 \div 15 \div 18^{\circ} \mathrm{C}$ d)

$9^{\circ} \mathrm{C}$
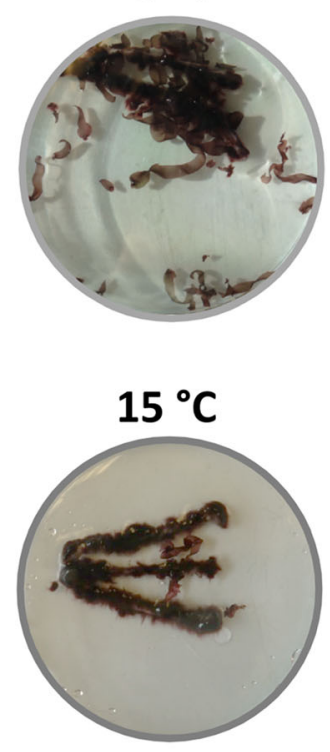

$18^{\circ} \mathrm{C}$

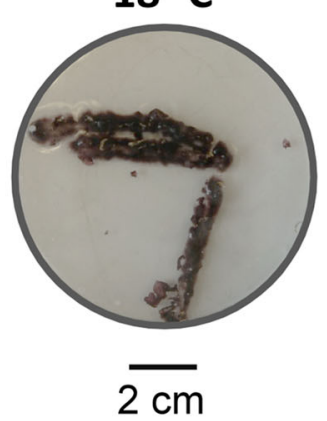

to fluctuating environmental conditions they are exposed to on the temperate rocky shores around the British Isles. However, these parameters varied in their importance and effect between different life history stages. Environmental conditions strongly influence zonation and survival in intertidal habitats (Colman 1933; Connell 1961; Somero 2002; Bird et al. 2013). The seasonal fluctuations in environmental conditions on temperate intertidal rocky shores results in the necessity of organisms to withstand wide temperature, irradiance and nutrient ranges (Lüning 1993). One adaptation of marine macrophytes to ensure survival in these highly dynamic and extreme habitats is a complex life cycle, with alterations in preferred conditions depending on the life history stage (Lubchenco and Cubit 1980; Cunningham et al. 1993). Changing environmental conditions can be important triggers in life history events such as initiating reproduction (Liu et al. 2017) and especially temperature and photoperiod have been found to be important factors in the life history of bladed Bangiales (Pereira et al. 2004; Lindstrom et al. 2008; LópezVivas et al. 2015).
Our results reflect the importance of different temperatures and photoperiods necessary for the life cycle completion of a temperate Porphyra species. Photoperiod and temperature were especially important in the development of the conchocelis phase. While a short or neutral photoperiod with high temperatures resulted in highest phyllospore germination success, a long photoperiod at $18{ }^{\circ} \mathrm{C}$ promoted best conchocelis growth. This suggests that the $P$. dioica conchocelis stage mainly grows during summer, when grazing pressure, temperatures and irradiances are high, and supports the hypothesis of the conchocelis stage being an escape strategy against unfavourable environmental conditions (Lubchenco and Cubit 1980). However, germination of phyllospores and growth of conchocelis at a lower temperature and a neutral or short photoperiod demonstrated that survival is not restricted to summer conditions. Porphyra dioica thalli with reproductive tissue occur on rocky shores of the Eastern Atlantic throughout the year but release of spores and germination into the conchocelis phase differs between seasons, peaking in spring (Holmes and Brodie 2004). We also 
a)

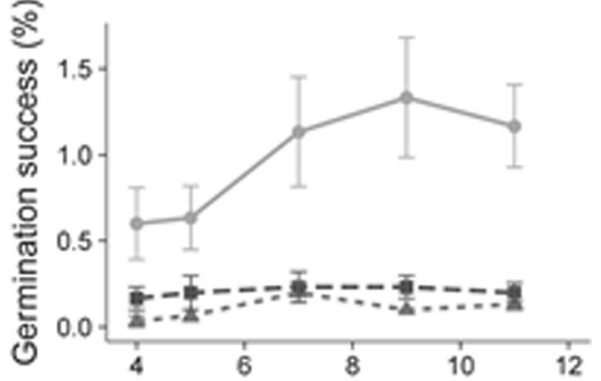

b)

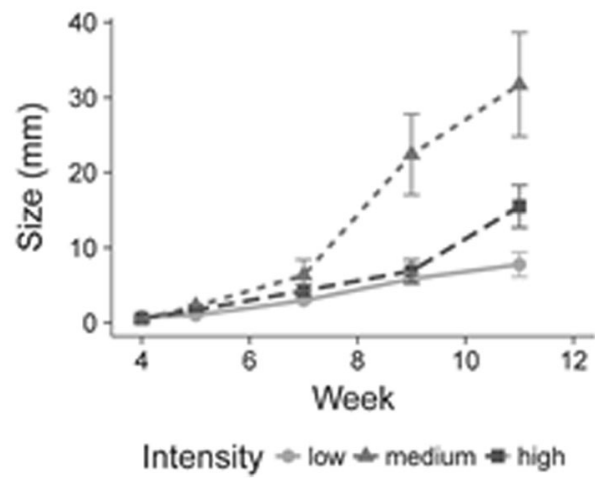

Fig. 7 The effect of light intensity (low $=80 \mu \mathrm{mol}$ photons $\mathrm{m}^{-2} \mathrm{~s}^{-1}$, medium $=130 \mu \mathrm{mol}$ photons $\mathrm{m}^{-2} \mathrm{~s}^{-1}$, high $=180 \mu \mathrm{mol}$ photons $\mathrm{m}^{-2} \mathrm{~s}^{-1}$ ) on Porphyra dioica conchospore germination success (Experiment 3 the "Effect of light intensity" section) (a) and size development (b) over an 11-week cultivation period at $15^{\circ} \mathrm{C}$ and a neutral photoperiod (12:12 h light:dark). The first measurable thalli were observed after 4 weeks after the experimental set up. Low light intensity enhanced conchospore germination $(p<0.05)$ while thalli size was largest at medium light intensity $(p<0.05)$. Displayed are averages \pm standard error

observed $P$. dioica blades year-round on intertidal rocky shores in South Wales (unpublished). Together with the results presented here, we assume that reproduction can occur all year but with a main reproduction and growth window that differs between life history stages.

The germination success of phyllospores into conchocelis in our experiments was low compared with Pereira et al. (2004) who observed P. dioica zygotospores germination of up to $80 \%$ at $20^{\circ} \mathrm{C}$, a neutral photoperiod with $25 \mu \mathrm{mol}$ photons $\mathrm{m}^{-2} \mathrm{~s}^{-1}$. However, initial spore densities used in their experiment were not stated. Therefore, it is likely that initial spore densities differed compared with our experiment, resulting in different germination success. Density is an important factor impacting recruitment success and growth in macroalgae (Reed 1990; Yang et al. 2006; Thirumaran and Anantharaman 2009). Further experiments are necessary to investigate the importance of initial spore density for maximal germination. Furthermore, the stress and desiccation induced phyllospore release in our experiment could have resulted in the release of premature spores which were not able to germinate, lowering the germination success. We investigated the reproductive tissue of thalli prior to the experiments but did not investigate the ratio of fertilised to unfertilised cells.
Therefore, another explanation for the low germination success could be a low fertilisation rate of the used tissue samples or that released spores were not mature. Additional studies are necessary to improve the germination success we observed in the experiments presented here.

In nature, the conchocelis stage (sporophyte) occurs in mollusc shells (Brodie and Irvine 2003) but is difficult to find because of its microscopic size. Asian Pyropia cultivation still relies on cultivating the conchocelis on oyster shells, even though research efforts have attempted to develop protocols for free-floating conchocelis (He and Yarish 2006; Li et al. 2011). Our results indicate that a shell substrate is not necessary for the development of the conchocelis stage and in fact, we even found a lower germination success of phyllospores on oyster shells. The lower germination success could be a result of spores slipping down from the smooth shell substrate or spores ending up beneath the shells, experiencing light availabilities that are too low to allow germination. Furthermore, colonies were larger growing on the shell substrate and it is possible that conchocelis grew overlapping each other as a result of closely germinating phyllospores. The larger colonies could also be a result of the colony morphology as conchocelis grew inside the shell matrix and appeared more spread compared with colonies growing on petri dishes. Conchocelis on Petri dishes were exposed to higher light intensities as the light was not filtered by the shell matrix and the more condensed growth form, in contrast to colonies grown on oyster shells, could be a protective response to higher light exposure since conchocelis are adapted to low light intensities (Lin et al. 2008). We could only account for colony diameter and not colony biomass, because conchocelis were growing within the oyster shell. It is therefore possible that colonies appeared larger as a result of the outspread morphology, but with equal growth and biomass compared with colonies grown on Petri dishes. Furthermore, the different colony morphologies demonstrated high phenotypic plasticity depending on the available substrate. High phenotypic plasticity has also been observed for the thallus phase and might be the result and explanation for the high success of bladed Bangiales surviving in the harsh conditions of intertidal rocky shore environments (Hannach and Waaland 1989). Lubchenco and Cubit (1980) discussed shell boring life history stages as an adaptation to elevated grazing pressure. Our findings support this hypothesis since phyllospores germinated and developed conchosporangia regardless of the substrate demonstrating that a shell substrate is not essential for survival and development. Furthermore, previous studies completing the life cycle of Pyropia (He and Yarish 2006; Li et al. 2011) and Porphyra (Pereira et al. 2004) species in suspension culture support the theory that the shell boring life phase is not essential but could be an escape of unfavourable environmental pressures.

Initiating the mass release of conchospores is the current bottleneck in European Porphyra cultivation limiting large- 
scale production. For the first time, we demonstrated the mass release of conchospores in a conchocelis suspension culture of $P$. dioica from the British Isles, induced by a drop in temperature. A sudden decrease in temperature has previously been shown to promote and initiate the release of conchospores from conchosporangia in bladed Bangiales (Mitman and van der Meer 1994; Orfanidis 2001; Pereira et al. 2004; Lindstrom et al. 2008). Successful conchospore release in $P$. dioica was previously only reported from Portugal (Pereira et al. 2004), where conchospores were released after a drop in temperature. However, released conchospore densities were not reported, limiting the comparability. Mass conchospore releases have been reported in bladed Bangiales, however, mainly in Pyropia species. He and Yarish (2006) reported a mass conchospore release of Pyropia leucosticta with 20 million conchospores per g DW of conchosporangia during peak conchospore release. In comparison, we observed lower conchospore densities of 1.5 million spores per g DW conchocelis culture after incubated at $9{ }^{\circ} \mathrm{C}$ for 5 days. However, the conchocelis culture used in our experiments constituted of a mixture of conchocelis and conchosporangia which could be a reason for the lower spore density observed compared with He and Yarish (2006). Additionally, amounts of released conchospores could be a species dependent trait. Conchospores have previously been observed in different Porphyra and Pyropia species to be released within a week if mature conchocelis cultures were transferred to favourable conditions (Chiang and Chou 1980; Kornmann and Sahling 1991; Orfanidis 2001). The timed mass conchospore release from free-floating conchocelis has important implications for possible commercial application. Initiating the mass release of conchospores from in suspension living conchocelis could increase space exploitation in contrast to the traditional method used throughout Asia where the conchocelis are still cultivated on a shell substrate. However, conchocelis grown on mollusc shells might be less prone to contamination by grazers, or overgrowth by diatoms, when grown in the shell matrix, facilitating an easier elimination or reduction of contamination by cleaning the shell surface.

We observed a high overgrowth of the cultivation ropes by conchocelis, especially under high temperatures and a long photoperiod. Since the germination success was low in all treatments, the question arose whether all spores developed into small thalli, or if the spore solution consisted partially of conchocelis archeospores which germinate into conchocelis (Nelson et al. 1999). Additionally, there is a possibility that small conchocelis fragments floating in the suspension culture were transferred with the conchospore solution. Following on these results, an effective and easy way of separating freefloating conchocelis filaments and conchospores, e.g. by centrifugation, should be evaluated.

The here presented results reveal the importance of environmental conditions for the development of the different life history stages of $P$. dioica. We demonstrated cultivation conditions for the successful life cycle completion of $P$. dioica and were able to initiate a mass conchospore release, a crucial part for the successful large-scale cultivation. However, environmental conditions for the successful life cycle completion can differ between and even within populations (Lindstrom et al. 2008). Therefore, the here identified conditions need to be validated in further strains from the same as well as from different $P$. dioica populations in order to develop a consistent cultivation protocol. Additionally, our results need to be considered with keeping the limitations of our experimental design in mind, especially in manipulating temperature. Furthermore, the low germination success of phyllospores and conchospores requires further investigation to find conditions that will result in a higher germination success.

Overall, we tackled one of the bottlenecks currently faced in European Porphyra cultivation. However, the cultivation is complex and time-consuming and will probably only be necessary if demand for Porphyra is increasing. Future research is needed to optimise the cultivation protocol and to investigate if these conditions stated here can be transferred for the successful life cycle completion in different $P$. dioica populations from the North East Atlantic.

\section{Summary and outlook}

Our results reflect the importance of environmental conditions, especially temperature, for the development of different $P$. dioica life history stages. While higher temperatures combined with a long photoperiod facilitate better growth in the conchocelis (sporophyte), a drop in temperature and a short photoperiod promote the transition to the thallus (gametophyte) phase. The successful induction of a mass conchospore release, of in suspension cultivated conchocelis, has important implications for possible commercial applications, to ensure a continuous supply of conchospores. However, further research is necessary to optimise settlement and germination success of conchospores to improve the low observed germination. Furthermore, these conditions need to be validated in further strains in order to address if required conditions for a successful cultivation show as much variability as demonstrated for other species. Nonetheless, this study moves the life cycle control of a European species one step further ahead, forming a basis on which to build cultivation.

Acknowledgements We would like to thank the team of the Centre for Sustainable Aquatic Research at Swansea University for their technical support.

Funding information The study was funded by a Knowledge Economy Skills Scholarship (KESS) - a pan-Wales higher level skills initiative led by Bangor University on behalf of the HE sector in Wales - partially funded by the Welsh Government's European Social Fund (ESF) 
convergence programme for West Wales and the Valleys and the Pembrokeshire Beachfood Company.

Open Access This article is distributed under the terms of the Creative Commons Attribution 4.0 International License (http:// creativecommons.org/licenses/by/4.0/), which permits unrestricted use, distribution, and reproduction in any medium, provided you give appropriate credit to the original author(s) and the source, provide a link to the Creative Commons license, and indicate if changes were made.

\section{References}

Abreu MH, Pereira R, Yarish C, Buschmann AH, Sousa-Pinto I (2011) IMTA with Gracilaria vermiculophylla: productivity and nutrient removal performance of the seaweed in a land-based pilot scale system. Aquaculture 312:77-87

Ashkenazi DY, Israel A, Abelson A (2018) A novel two-stage seaweed integrated multi-trophic aquaculture. Rev Aquac 11:246-262

Bird CE, Franklin EC, Smith CM, Toonen RJ (2013) Between tide and wave marks: a unifying model of physical zonation on littoral shores. PeerJ 1:e154

Blouin NA, Brodie JA, Grossman AC, Xu P, Brawley SH (2011) Porphyra: a marine crop shaped by stress. Trends Plant Sci 16:2937

Brodie JA, Irvine LM (2003) Seaweeds of the British Isles - volume 1 Rhodophyta - part 3B Bangiophycidae. Intercept Limited, Hampshire

Burtin P (2003) Nutritional value of seaweeds. Electron J Environ Agric Food Chem 2:498-503

Capuzzo E, McKie T (2016) Seaweed in the UK and abroad - status, products, limitations, gaps and Cefas role. Cefas Contract Report FC002I. Cefas, Lowestoft, p 66

Chiang Y-M, Chou Y-H (1980) The free conchocelis of Porphyra angusta and Porphyra dentata 1. Influence of light and temperature on the maturation of conchosporangia and conchospore liberation. Natl Sci Counc Mon 8:323-328

Chopin T, Buschmann AH, Halling C, Troell M, Kautsky N, Neori A, Kraemer GP, Zertuche-González JA, Yarish C, Neefus C (2001) Integrating seaweeds into marine aquaculture systems: a key toward sustainability. J Phycol 37:975-986

Chung IK, Kang YH, Yarish C, Kraemer GP, Lee JA (2002) Application of seaweed cultivation to the bioremediation of nutrient-rich effluent. Algae 17:187-194

Cian RE, Fajardo MA, Alaiz M, Vioque J, González RJ, Drago SR (2014) Chemical composition, nutritional and antioxidant properties of the red edible seaweed Porphyra columbina. Int J Food Sci Nutr 65: 299-305

Cian RE, Drago SR, De Medina FS, Martínez-Augustin O (2015) Proteins and carbohydrates from red seaweeds: evidence for beneficial effects on gut function and microbiota. Mar Drugs 13:53585383

Colman J (1933) The nature of the intertidal zonation of plants and animals. J Mar Biol Assoc U K 18:435-476

Connell JH (1961) The influence of interspecific competition and other factors on the distribution of the barnacle Chthamalus stellatus. Ecology 42:710-723

Cunningham EM, Guiry MD, Breeman AM (1993) Environmental regulation of development, life history and biogeography of Helminthora stackhousei (Rhodophyta) by daylength and temperature. J Exp Mar Biol Ecol 171:1-21
Dhargalkar VK, Pereira N (2005) Seaweed: promising plant of the millennium. Sci Cult 71:60-66

Drew KM (1949) Conchocelis-phase in the life-history of Porphyra umbilicalis (L.) Kütz. Nature 164:748-749

Dring MJ (1967) Effect of daylength on growth and reproduction of the conchocelis-phase of Porphyra tenera. J Mar Biol Assoc U K 47: $501-510$

FAO (2018) The state of world fisheries and aquaculture 2018 - meeting the sustainable development goals. FAO, Rome Public Output report of the EnAlgae project, Swansea, pp36 June 2016, Available online at http://www.enalgae.eu

Golven P, Le Goff T, Champenois J (2014) Seeding techniques used at CEVA for cultivation of Saccharina latissima Public Output report of the EnAlgae project, Swansea, June 2016, 36pp, Available online at http://www.enalgae.eu

Green LA, Neefus CD (2015) Effects of temperature, light level, photoperiod, and ammonium concentration on Pyropia leucosticta (Bangiales, Rhodophyta) from the Northwest Atlantic. J Appl Phycol 27:1253-1261

Hannach G, Waaland JR (1989) Growth and morphology of young gametophytes of Porphyra abbottae (Rhodophyta): effects of environmental factors in culture. J Phycol 25:247-254

He P, Yarish C (2006) The developmental regulation of mass cultures of free-living conchocelis for commercial net seeding of Porphyra leucosticta from Northeast America. Aquaculture 257:373-381

He P, Xu S, Zhang H, Wen S, Dai Y, Lin S, Yarish C (2008) Bioremediation efficiency in the removal of dissolved inorganic nutrients by the red seaweed, Porphyra yezoensis, cultivated in the open sea. Water Res 42:1281-1289

Holmes MJ, Brodie J (2004) Morphology, seasonal phenology and observations on some aspects of the life history in culture of Porphyra dioica (Bangiales, Rhodophyta) from Devon, UK. Phycologia 43: 176-188

Holmes MJ, Brodie J (2005) Phenology and the life history in culture of Porphyra leucosticta (Bangiales, Rhodophyta) from Britain. Bot Mar 48:218-230

Kornmann P, Sahling PH (1991) The Porphyra species of Helgoland (Bangiales, Rhodophyta). Helgol Meeresunters 45:1-38

Kraemer GP, Carmona R, Neefus C, Chopin T, Miller S, Tang X, Yarish C (2004) Preliminary examination of the bioremediation and mariculture potential of a Northeast U. S. A. and an Asian species of Porphyra. Bull Fish Res Agency 1:77-82

Levine IA, Sahoo D (2010) Porphyra - harvesting gold from the sea. I.K. International Publishing House Pvt. Ltd., New Delhi

Li X, Yang L, He P-M (2011) Formation and growth of free-living conchosporangia of Porphyra yezoensis: effects of photoperiod, temperature and light intensity. Aquac Res 42:1079-1086

Lin R, Lindstrom SC, Stekoll MS (2008) Photosynthesis and respiration of the conchocelis stage of Alaskan Porphyra (Bangiales, Rhodophyta) species in response to environmental variables. J Phycol 44:573-583

Lindstrom SC, Conitz JM, Hall S, Stekoll MS (2008) Induction of conchospore release: Ecotypic variation in northeast Pacific species of Porphyra. J Appl Phycol 20:331-340

Liu X, Bogaert K, Engelen AH, Leliaert F, Roleda MY, De Clerck O (2017) Seaweed reproductive biology: environmental and genetic controls. Bot Mar 60:89-108

López-Vivas JM, Riosmena-Rodríguez R, de la Llave AAJ-G, PachecoRuíz I, Yarish C (2015) Growth and reproductive responses of the conchocelis phase of Pyropia hollenbergii (Bangiales, Rhodophyta) to light and temperature. J Appl Phycol 27:1561-1570

$\mathrm{Lu} \mathrm{S}$, Yarish C (2011) Interaction of photoperiod and temperature in the development of conchocelis of Porphyra purpurea (Rhodophyta: Bangiales). J Appl Phycol 23:89-96 
Lubchenco J, Cubit J (1980) Heteromorphic life histories of certain marine algae as adaptations to variations in herbivory. Ecology 61:676687

Lüning K (1993) Environmental and internal control of seasonal growth in seaweeds. Hydrobiologia 260/261:1-14

Mac Monagail M, Cornish L, Morrison L, Araújo R, Critchley AT (2017) Sustainable harvesting of wild seaweed resources. Eur J Phycol 52: 371-390

Mahadevan K (2015) Seaweeds: a sustainable food source. In: Tiwari BK, Troy DJ (eds) Seaweed sustainability - food and non-food applications. Elsevier, Amsterdam, pp 347-364

Markham JW, Hagmeier E (1982) Observations on the effects of germanium dioxide on the growth of macro-algae and diatoms. Phycologia $21: 125-130$

Mitman GG, van der Meer JP (1994) Meiosis, blade development, and sex determination in Porphyra purpurea (Rhodophyta). J Phycol 30:147-159

Nelson W, Brodie J, Guiry M (1999) Terminology used to describe reproduction and life history stages in the genus Porphyra (Bangiales, Rhodophyta). J Appl Phycol 11:407-410

Orfanidis S (2001) Culture studies of Porphyra leucosticta (Bangiales, Rhodophyta) from the Gulf of Thessaloniki, Greece. Bot Mar 44: 533-539

Organic Monitor (2014) The European market for sea vegetables introduction. http://www.bim.ie/media/bim/content/publications/ The,European,Market,for,Sea,Vegetables,-,2015.pdf

Ortiz J, Romero N, Robert P, Araya J, Lopez-Hernández J, Bozzo C, Navarrete E, Osorio A, Rios A (2006) Dietary fiber, amino acid, fatty acid and tocopherol contents of the edible seaweeds Ulva lactuca and Durvillaea antarctica. Food Chem 99:98-104

Pereira R, Sousa-Pinto I, Yarish C (2004) Field and culture studies of the life history of Porphyra dioica (Bangiales, Rhodophyta) from Portugal. Phycologia 43:756-767

Pereira R, Yarish C, Sousa-Pinto I (2006) The influence of stocking density, light and temperature on the growth, production and nutrient removal capacity of Porphyra dioica (Bangiales, Rhodophyta). Aquaculture 252:66-78

Pereira R, Kraemer G, Yarish C, Sousa-Pinto I (2008) Nitrogen uptake by gametophytes of Porphyra dioica (Bangiales, Rhodophyta) under controlled-culture conditions. Eur J Phycol 43:107-118

Pinheiro J, Bates D, DebRoy S, Sarkar D, Team RC (2019) Nlme: linear and nonlinear mixed effects models. R package version 3.1-139. R Core Team, Vienna

Reed DC (1990) An experimental evaluation of density dependence in a subtidal algal population. Ecology 71:2286-2296
Sidirelli-Wolff M (1992) The influence of temperature, irradiance and photoperiod on the reproductive life history of Porphyra leucosticta (Bangiales, Rhodophyta) in laboratory culture. Bot Mar 35:251-257

Somero GN (2002) Thermal physiology and vertical zonation of intertidal animals: optima, limits, and costs of living. Integr Comp Biol 42: 780-789

Stagnol D, Renaud M, Davoult D (2013) Effects of commercial harvesting of intertidal macroalgae on ecosystem biodiversity and functioning. Estuar Coast Shelf Sci 130:99-110

Thirumaran G, Anantharaman P (2009) Daily growth rate of field farming seaweed Kappaphycus alvarezii (Doty) Doty ex P. Silva in Vellar Estuary. World J Fish Mar Sci 1:144-153

Turner NJ (2003) The ethnobotony of edible seaweed (Porphyra abbottae and related species; Rhodophyta: Bangiales) and its use by First Nations on the Pacific coast of Canada. Can J Bot 81: 283-293

Varela-Alvarez E, Stengel D, Guiry M (2004) The use of image processing in assessing conchocelis growth and conchospore production in Porphyra linearis. Phycologia 42:282-287

Varela-Álvarez E, Loureiro J, Paulino C, Serrão EA (2018) Polyploid lineages in the genus Porphyra. Sci Rep 8:1-15

Walsh M, Watson L (2012) Towards the further development of seaweed aquaculture in Ireland

Wang J, Dai J, Zhang Y (2006) Nuclear division of the vegetative cells, conchosporangial cells and conchospores of Porphyra yezoensis (Bangiales, Rhodophyta). Phycol Res 54:201-207

Yan X, He L, Aruga Y (2007) Karyological observations on the occurrence of meiosis in the life cycle of Porphyra haitanensis Chang et Zheng (Bangiales, Rhodophyta). Bot Mar 50:257-263

Yang YF, Fei XG, Song JM, Hu HY, Wang GC, Chung IK (2006) Growth of Gracilaria lemaneiformis under different cultivation conditions and its effects on nutrient removal in Chinese coastal waters. Aquaculture 254:248-255

Yong YS, Yong WTL, Anton A (2013) Analysis of formulae for determination of seaweed growth rate. J Appl Phycol 25:1831-1834

Zhong Z, Wang W, Sun X, Liu F, Liang Z, Wang F, Chen W (2016) Developmental and physiological properties of Pyropia dentata (Bangiales, Rhodophyta) conchocelis in culture. J Appl Phycol 28: 3435-3445

Publisher's note Springer Nature remains neutral with regard to jurisdictional claims in published maps and institutional affiliations. 